\title{
ON THE REGULARITY OF GENERALIZED CONVEX FUNCTIONS $\left(^{1}\right)$
}

\author{
BY

\section{J. H. B. KEMPERMAN}

1. Introduction. If a measurable or locally bounded function $f$ satisfies sufficiently many inequalities then often it happens that $f$ has automatically much stronger regularity properties such as continuity or differentiability.

We shall study this type of problem in detail in $\S 3-6$ for the property of $f$ being (generalized) convex relative to a given (nonlinear) $n$-parameter family $\mathscr{F}_{n}$ of functions. The corresponding results are summarized in $\$ 2$.

As a somewhat different problem, we shall investigate in $\S 7$ the type of regularity possessed by all measurable solutions of the inequality

$$
\sum_{j=0}^{n} a_{j} f\left(x+T_{j} y\right) \geqq 0 .
$$

2. Summary. Let $(a, b)=\{x: a<x<b\}$ be a given finite open interval in $R$. Following the terminology of Popoviciu [19], a given function $f$ on $(a, b)$ will be called $(n-1)$-convex (or $(n-1)$-convex in the sense of Jensen) if

$$
\Delta_{h}^{n} f(x)=\sum_{j=0}^{n}(-1)^{n-j}\left(\begin{array}{l}
n \\
j
\end{array}\right) f(x+j h) \geqq 0
$$

for each choice of the numbers $h>0$ and $a<x<b-n h$. Here, $n$ denotes a fixed integer with $n \geqq 2$; (for $n=1,(2.1)$ merely says that $f$ is nondecreasing on $(a, b)$ ). The following result is due to Ciesielski [3].

(2.2) THEOREM. Let $f$ be an $(n-1)$-convex function on $(a, b)$ such that $f$ is bounded on at least one measurable subset $A$ of $(a, b)$ having a positive measure. Then $f$ is continuous on $(a, b)$.

Here and further on, the notions of measure and measurability are always to be interpreted in the sense of Lebesgue. It would not be enough, in Theorem 2.2, that $f$ be bounded below on $(a, b)$ as follows from the example $f(x)=\phi(x)^{2}$ with $\phi$ as a real-valued nonmeasurable function which is additive in the sense that $\phi(x+y)=\phi(x)+\phi(y)$ for all $x, y \in R$. If $n \geqq 3$ it would not be enough that $f$ be bounded above on $(a, b)$, since $f(x)=-\phi(x)^{2}$ satisfies $\Delta_{h}^{3} f(x) \equiv 0$ for any $h$. However, as was shown by Ostrowski [13], if $f$ is 1-convex $(n=2)$ and bounded above on a measurable set of positive measure then $f$ is continuous.

Received by the editors July $31,1967$.

${ }^{(1)}$ This research was supported in part by the National Science Foundation Grant GP-7353. 
If $x_{1}, \ldots, x_{n}$ are distinct points in $(a, b)$, let us denote by $L(x)=L\left(x_{1}, \ldots, x_{n}\right.$; $f \mid x)$ the unique polynomial of degree $\leqq n-1$ such that $L\left(x_{1}\right)=f\left(x_{1}\right), \ldots, L\left(x_{n}\right)$ $=f\left(x_{n}\right)$. As is easily seen, property (2.1) may be rewritten as

$$
L\left(x_{0}, x_{0}+h, \ldots, x_{0}+(n-1) h ; f \mid x_{0}+n h\right) \leqq f\left(x_{0}+n h\right),
$$

holding when $h>0$ and $a<x_{0}<b-n h$. The above can be generalized by replacing the family of all ordinary polynomials of degree $\leqq n-1$ by a different family.

In the sequel all functions will be taken to be real-valued. Further, $n$ denotes a fixed integer with $n \geqq 2$.

(2.4) Definition. By an $n$-parameter family $\mathscr{F}_{n}$ on $(a, b)$ we shall mean a collection $\mathscr{F}_{n}$ of continuous functions on $(a, b)$ such that for every choice of the $n$ distinct points $x_{1}, \ldots, x_{n}$ in $(a, b)$ and every choice of the $n$ real numbers $y_{1}, \ldots, y_{n}$ there exists a unique member $L \in \mathscr{F}_{n}$ satisfying

$$
L\left(x_{1}\right)=y_{1}, \ldots, L\left(x_{n}\right)=y_{n} .
$$

In particular, two distinct members $g_{1}, g_{2}$ of $\mathscr{F}_{n}$ cannot be equal at more than $n-1$ distinct points.

Following Moldovan [10], the above unique function $L \in \mathscr{F}_{n}$ will be denoted as

$$
L\left(x_{1}, \ldots, x_{n} ; y_{1}, \ldots, y_{n} \mid x\right) \text {. }
$$

We further write

$$
L\left(x_{1}, \ldots, x_{n} ; f \mid x\right)=L\left(x_{1}, \ldots, x_{n} ; f\left(x_{1}\right), \ldots, f\left(x_{n}\right) \mid x\right) .
$$

Usually, we take the $x_{i}$ such that $a<x_{1}<\cdots<x_{n}<b$. In the sequel, unless otherwise stated, $\mathscr{F}_{n}$ will be understood to be a given $n$-parameter family in $(a, b)$.

(2.6) Definition. A function $f$ on $(a, b)$ will be said to be weakly $\mathscr{F}_{n}$-convex (or $\mathscr{F}_{n}$-convex in the sense of Jensen) if the inequality (2.3) holds for each choice of the numbers $h$ and $x_{0}$ such that $h>0$ and $a<x_{0}<b-n h$.

The following result will be proved in $\S 4$. It is a generalization of the above Theorem 2.2 due to Ciesielski.

(2.7) THEOREM. Let the function $f$ on $(a, b)$ be weakly $\mathscr{F}_{n}$-convex. Suppose further that $f$ is bounded on a measurable subset $A$ of $(a, b)$ of positive measure. Then $f$ is continuous on $(a, b)$.

The case $n=2$ of Theorem 2.7 is due to Beckenbach and Bing [1]. Actually, they only required that $f$ be bounded above on $A$, thus generalizing a previously mentioned result of Ostrowski [13].

If one is willing to assume that $f$ is measurable then the condition that $f$ be weakly $\mathscr{F}_{n}$-convex can be replaced by an even weaker condition. For a precise statement, see Theorem 7.3.

(2.8) The following result gives some insight in the structure of an arbitrary nonmeasurable weakly $\mathscr{F}_{n}$-convex function. The special case that $k=1$ and $n=2$ is due to Beckenbach and Bing [1]. 
(2.9) THEOREM. Let $f$ be a given weakly $\mathscr{F}_{n}$-convex function on $(a, b)$. Let further $x_{0}$ and $h_{1}, \ldots, h_{k}$ be given real numbers. Then there exists a unique continuous function $g\left(z_{1}, \ldots, z_{k}\right)$ on the strip $a<x_{0}+\sum h_{j} z_{j}<b$ in $R^{k}$ such that

$$
f\left(x_{0}+r_{1} h_{1}+\cdots+r_{k} h_{k}\right)=g\left(r_{1}, r_{2}, \ldots, r_{k}\right)
$$

holds for each choice of the rational numbers $r_{1}, \ldots, r_{k}$ for which

$$
a<x_{0}+\sum r_{j} h_{j}<b .
$$

(2.11) Definition. A function $f$ on $(a, b)$ is said to be $\mathscr{F}_{n}$-convex (or also strongly $\mathscr{F}_{n}$-convex) if

$$
L\left(x_{1}, \ldots, x_{n} ; f \mid x_{n+1}\right) \leqq f\left(x_{n+1}\right)
$$

holds whenever $a<x_{1}<\cdots<x_{n}<x_{n+1}<b$.

The property of $\mathscr{F}_{n}$-convexity was already studied by Tornheim [22], Hartman [6], Morozov [12] and Moldovan [10], and for certain linear families $\mathscr{F}_{n}$ by Popoviciu [17], [18], [19], [20], Pólya [15], Karlin and Studden [7, pp. 375-466], and many others; see also the study [24] of the associated linear differential operators.

(2.13) LEMma. In order that a function $f$ on $(a, b)$ be $\mathscr{F}_{n}$-convex, it is necessary and sufficient that $f$ be both continuous and weakly $\mathscr{F}_{n}$-convex.

(2.14) Let $C\left(\mathscr{F}_{n}\right)$ denote the class of all (strongly) $\mathscr{F}_{n}$-convex functions on $(a, b)$. Observe that (2.12) holds with the equality sign when $f \in \mathscr{F}_{n}$, implying that

$$
\mathscr{F}_{n} \subset C\left(\mathscr{F}_{n}\right) \text {. }
$$

Let us now turn to the question what other regularity properties (besides continuity) are satisfied by all $\mathscr{F}_{n}$-convex functions. Let $\Lambda$ stand for any class of continuous functions on $(a, b)$ such that $\phi \notin \Lambda$ for at least one continuous function $\phi$. By (2.15), in order that $C\left(\mathscr{F}_{n}\right) \subset \Lambda$ it is at least necessary that $\mathscr{F}_{n} \subset \Lambda$. Considering the class $\mathscr{F}_{n}^{\prime}=\left\{f^{\prime}=f+\psi, f \in \mathscr{F}_{n}\right\}$ with $\psi$ fixed, we see that $\mathscr{F}_{n} \subset \Lambda$ can never be a consequence of the fact that $\mathscr{F}_{n}$ is an $n$-parameter family. The best one can hope for is an implication of the type $\mathscr{F}_{n} \subset \Lambda \Rightarrow C\left(\mathscr{F}_{n}\right) \subset \Lambda$, with or without some weak additional condition imposed on $\mathscr{F}_{n}$. Let us first review some known results in this direction.

(2.16) Let $x_{0} \in(a, b)$ be fixed. It was shown by Tornheim [22, p. 462], that each $f \in C\left(\mathscr{F}_{n}\right)$ is differentiable at $x_{0}$ as soon as each $f \in \mathscr{F}_{n}$ is differentiable at $x_{0}$ and, moreover, $n \geqq 3$.

(2.17) This result was generalized by Hartman [6] as follows. Let $x_{0} \in(a, b)$ and let $k \geqq 0$ denote an integer. Denote by $\Gamma^{k}\left(x_{0}\right)$ the class of continuous functions $f$ on $(a, b)$ having at $x_{0}$ a Taylor derivative of order $k$, in the sense that

$$
f(x)=\sum_{i=0}^{k} f^{i}\left(x_{0}\right)\left(x-x_{0}\right)^{i}+o\left(x-x_{0}\right)^{k}
$$

as $x$ tends to $x_{0}$. Here, $f^{i}\left(x_{0}\right)(i=0, \ldots, k)$ denote constants. 
The function $f(x)=x^{2+\varepsilon} \sin x^{-\varepsilon}(\varepsilon>0)$ has at $x=0$ a second Taylor derivative but the ordinary second derivative $f^{\prime \prime}(0)$ does not exist. A stronger counterexample of this kind may be found in [4, p. 277].

We shall say that a family $\mathscr{F}_{n}$ has property $\Gamma_{0}^{k}\left(x_{0}\right)$ if $\mathscr{F}_{n} \subset \Gamma^{k}\left(x_{0}\right)$. We say that $\mathscr{F}_{n}$ has property $\Gamma_{1}^{k}\left(x_{0}\right)$ if, moreover, for each choice of the real numbers $c_{0}, \ldots, c_{k}$ there is at least one $f \in \mathscr{F}_{n}$ such that

$$
f^{i}\left(x_{0}\right)=c_{i}, \quad(i=0,1, \ldots, k) .
$$

Finally, let us say that $\mathscr{F}_{n}$ has property $\Gamma_{2}^{k}\left(x_{0}\right)$ if, moreover, two distinct solutions $f_{1}, f_{2} \in \mathscr{F}_{n}$ of (2.19) cannot be equal at more than $n-k-2$ distinct points $x \neq x_{0}$. For $k=0$ these properties always hold.

Hartman [6, p. 138, p. 140], proved the following. Let $1 \leqq k \leqq n-2$ and $x_{0} \in(a, b)$ be fixed. Then $C\left(\mathscr{F}_{n}\right) \subset \Gamma^{k}\left(x_{0}\right)$ as soon as $\mathscr{F}_{n}$ has both properties $\Gamma_{0}^{k}\left(x_{0}\right)$ and $\Gamma_{2}^{k-1}\left(x_{0}\right)$. Next assume that $\mathscr{F}_{n}$ has both properties $\Gamma_{1}^{n-1}\left(x_{0}\right)$ and $\Gamma_{2}^{n-2}\left(x_{0}\right)$. Then each $f \in$ $C\left(\mathscr{F}_{n}\right)$ has at $x_{0}$ a Taylor derivative of order $n-2$ and, moreover, one-sided Taylor derivatives of order $n-1$ (in an obvious sense). In the special case $n=2$ the latter result says that each $f \in C\left(\mathscr{F}_{n}\right)$ has one-sided derivatives at $x_{0}$ as soon as $\mathscr{F}_{n}$ satisfies. property $\Gamma_{1}^{1}\left(x_{0}\right)$. For a related result, see Peixoto [14].

(2.20) Let us briefly consider the important special case where $\mathscr{F}_{n}$ is a linear family. Here, $\mathscr{F}_{n}$ consists of all linear combinations

$$
f=c_{1} u_{1}+\cdots+c_{n} u_{n}
$$

of a given set $\left\{u_{1}, \ldots, u_{n}\right\}$ of $n$ linearly independent continuous functions on $(a, b)$. The condition (2.4) that $\mathscr{F}_{n}$ be an $n$-parameter family now can be expressed as the condition that the determinant

$$
U=\operatorname{det}\left(\begin{array}{c}
u_{1}, \ldots, u_{n} \\
x_{1}, \ldots, x_{n}
\end{array}\right)=\operatorname{det}\left(u_{i}\left(x_{j}\right)\right)
$$

be nonzero whenever $x_{1}<x_{2}<\cdots<x_{n}$. By continuity, one may as well assume that $U>0$ whenever $x_{1}<\cdots<x_{n}$.

In the present special case, $L(x)=L\left(x_{1}, \ldots, x_{n} ; f \mid x\right)$ admits the explicit formula

$$
\operatorname{det}\left(\begin{array}{l}
u_{1}, \ldots, u_{n}, f \\
x_{1}, \ldots, x_{n}, x
\end{array}\right)=(f(x)-L(x)) U .
$$

Hence, the function $f$ on $(a, b)$ is $\mathscr{F}_{n}$-convex if and only if the determinant (2.22) is nonnegative whenever $x_{1}<\cdots<x_{n}<x$.

(2.23) Let us now impose the assumption that each $u_{i}$ has a continuous $(n-1)$ th derivative $u_{i}^{(n-1)}$ and that, moreover,

$$
\operatorname{det}\left(u_{i}^{(j-1)}(x) ; i, j=1, \ldots, k\right)>0,
$$

for all $k=1, \ldots, n,(a<x<b)$. In particular, $u_{1}(x)>0$. Under this assumption, and 
provided $u_{1}, \ldots, u_{n}$ and $f$ have $n$ derivatives, it follows from (2.22) and a result of Pólya [15], see [16, p. 55], that $f$ is $\mathscr{F}_{n}$-convex if and only if

$$
\operatorname{det}\left(u_{i}^{(j-1)}(x) ; i, j=1, \ldots, n+1\right) \geqq 0
$$

for all $a<x<b$; here, $u_{n+1}=f$.

The differential inequality (2.25) generalizes the classical condition $f^{\prime \prime} \geqq 0$ for (ordinary) convexity relative to the 2-parameter family of all linear functions $f(x)=c x+d$.

Popoviciu [18] proved (under the assumption (2.24)) that every $\mathscr{F}_{n}$-convex function $f$ has an absolutely continuous $(n-2)$ th derivative, hence, the derivative $f^{(n-1)}(x)$ exists almost everywhere. He proved this by showing that, locally, $f$ has a bounded $(n-1)$ th divided difference, compare [11, p. 183].

For this same case, Karlin and Studden [7, p. 381, p. 462], established that an $\mathscr{F}_{n}$-convex function $f$ does have everywhere a right continuous $(n-1)$ th right derivative $f^{(n-1)}(x+0)$. Moreover, a specific expression $\sum_{j=0}^{n-1} a_{j}(x) f^{(j)}(x+0)$ (having (2.25) as its "derivative") is shown to be nondecreasing. Similarly for the left derivatives.

Actually, it would be largely sufficient to assume (2.24) only for $k=n$. For, afterwards, one can always at least locally achieve the full property (2.24) by replacing the $u_{i}$ by suitable linear combinations $u_{i}^{*}=\sum_{j} c_{i j} u_{j}$.

Let us now state some new results. Here, $C_{k}$ denotes the class of real-valued functions on $(a, b)$ having everywhere a $k$ th derivative. Further, $\mathscr{F}_{n}$ is again an arbitrary (nonlinear) $n$-parameter family as in (2.4).

(2.26) Definition. Let $k$ be a positive integer. We shall denote by $D_{k}$ the class of all $f \in C_{k-1}$ such that the derivative $f^{(k-1)}$ is of the form

$$
f^{(k-1)}(x)=f^{(k-1)}\left(x_{0}\right)+\int_{x_{0}}^{x} \phi(t) d t, \quad(a<x<b) .
$$

Here, the function $\phi$ on $(a, b)$ is assumed to be locally bounded with at most denumerably many discontinuities, all these of the first kind.

In particular, $\phi(t-0)$ and $\phi(t+0)$ exist everywhere and, moreover, are equal outside some finite or denumerable set $D(\phi)$. It follows that each $f \in D_{k}$ has everywhere a left continuous left $k$ th derivative $f^{(k)}(x-0)=\phi(x-0)$ and everywhere a right continuous right $k$ th derivative $f^{(k)}(x+0)=\phi(x+0)$. Finally, the $k$ th derivative $f^{(k)}(x)$ exists at each point $x$ outside the above denumerable set $D(\phi)$.

(2.28) Definition. We shall say that the $n$-parameter family $\mathscr{F}_{n}$ satisfies condition $C_{k}^{*}$ if each $f \in \mathscr{F}_{n}$ has a continuous $k$ th derivative, in such a way that the function

$$
L^{(k)}\left(y_{1}, \ldots, y_{n} \mid x\right)=\left(\frac{d}{d x}\right)^{k} L\left(z_{1}, \ldots, z_{n} ; y_{1}, \ldots, y_{n} \mid x\right)
$$

is jointly continuous in the $n+1$ variables $y_{i} \in R, x \in(a, b)$, whenever $z_{1}<\cdots<z_{n}$ are fixed. 
Actually, the latter property for one fixed set of $z_{i}$ is easily seen to imply the same property for any other such set. In the linear case (2.21) condition $C_{k}^{*}$ is satisfied as soon as each of the $n$ functions $u_{i}$ has a continuous $k$ th derivative.

The proof of the following result will be given in $\$ 6$. For a related result, see Theorem 7.18.

(2.30) THEOREM. Let $1 \leqq k \leqq n-1$ be a fixed integer. Let $\mathscr{F}_{n}$ be an arbitrary $n$-parameter family in $(a, b)$ and suppose that $\mathscr{F}_{n}$ satisfies the above condition $C_{k}^{*}$. Then we have for each $\mathscr{F}_{n}$-convex function $f$ on $(a, b)$ that $f \in D_{k}$; in particular, the derivative $f^{(k)}(x)$ exists at all but denumerably many points.

3. Auxiliary results. Let $n \geqq 2$ and let $\mathscr{F}_{n}$ be an arbitrary $n$-parameter family in $(a, b)$. The following result due to Tornheim [22, p. 460], will turn out to be an extremely useful result.

(3.1) THEOREM. In its domain the function

$$
L\left(x_{1}, \ldots, x_{n} ; y_{1}, \ldots, y_{n} \mid x\right)
$$

is jointly continuous in the $2 n+1$ variables $x_{i}, y_{i}$ and $x$.

Here, the domain may be defined by $y_{i} \in R$ and

$$
a<x_{1}<\cdots<x_{n}<b, \quad a<x<b .
$$

It follows that the continuity of the above $L$-function, regarded as a function of $x$ and $x_{1}, \ldots, x_{n}\left(a<x_{1}<\cdots<x_{n}<b, a<x<b\right)$, is at each point uniform with respect to the "parameter" $y=\left(y_{1}, \ldots, y_{n}\right)$ as long as $y$ is restricted to a bounded subset of $R^{n}$. We further conclude that $L$ is uniformly bounded as long as $y$ is restricted to a bounded set and $x_{i+1}-x_{i} \geqq \delta\left(i=0,1, \ldots, n+1 ; x_{0}=a, x_{n+1}=b\right)$, $a+\delta \leqq x \leqq b-\delta$. Here, $\delta$ denotes an arbitrary positive constant.

As an application of Theorem 3.1, we have the following result due to Morozov $[12$, p. 83].

(3.2) LEMMA. Let $f_{1}$ and $f_{2}$ be fixed distinct members of $\mathscr{F}_{n}$. Let $k$ denote the number of distinct zeros of $f_{2}-f_{1}$ and let $k^{\prime} \leqq k$ denote the number of those zeros near which $f_{2}-f_{1}$ does not change sign (a so-called loop root).

Then $k+k^{\prime} \leqq n-1$. In particular, if $f_{2}-f_{1}$ has $n-1$ zeros then $f_{2}-f_{1}$ must change sign at each of these zeros.

Proof. Clearly, $k \leqq n-1<\infty$. Choose $f_{3} \in \mathscr{F}_{n}$ such that:

(i) $f_{3}(x)=f_{2}(x)$ at the $k-k^{\prime}$ ordinary zeros of $f_{2}-f_{1}$,

(ii) $f_{3}(x)=f_{2}(x) \pm \varepsilon$ at the $k^{\prime}$ loop roots of $f_{2}-f_{1}$,

(iii) $f_{3}(x)=f_{2}(x)$ at $n-k$ further points.

By Theorem 3.1, choosing $\varepsilon>0$ sufficiently small, we can make the maximum deviation of $f_{2}$ and $f_{3}$ (in any compact interval) as small as we please. Choosing moreover the \pm signs according to the local sign of $f_{1}-f_{2}$, one attains a situation where $f_{3}-f_{1}$ has at least $\left(k-k^{\prime}\right)+2 k^{\prime}=k+k^{\prime}$ zeros; hence, $k+k^{\prime} \leqq n-1$. 
(3.3) In the following Lemma 3.6, $f$ denotes a fixed function on $(a, b)$ while $S$ denotes a fixed set of $n+1$ points $x_{i}$ ordered in such a way that $a<x_{1}<\cdots<x_{n}$ $<x_{n+1}<b$. Further, for $i=1, \ldots, n, n+1$, put

$$
g_{i}(x)=L\left(x_{1}, \ldots, x_{i-1}, x_{i+1}, \ldots, x_{n+1} ; f \mid x\right)
$$

and

$$
D_{i}=D_{i}\left(x_{1}, \ldots, x_{n+1} ; f\right)=f\left(x_{i}\right)-g_{i}\left(x_{i}\right) .
$$

(3.6) LEMma. If $D_{j}=0$ for one value $j$ then $g_{i}(x) \equiv g_{j}(x)$ and $D_{i}=0$ for all $i$. Suppose not. Then the $D_{i}$ are of alternating sign

$$
\operatorname{sgn}\left(D_{i}\right)=(-1)^{n+1-i} \varepsilon, \quad(i=1, \ldots, n+1),
$$

where $\varepsilon=\operatorname{sgn}\left(D_{n+1}\right)= \pm 1$. Moreover, if $1 \leqq i<j \leqq n+1$ then $g_{i}(x)-g_{j}(x) \neq 0$ outside $S$ in such a way that

$$
\begin{aligned}
\operatorname{sgn}\left\{g_{i}(x)-g_{j}(x)\right\} & =\varepsilon & & \text { if } x>x_{n+1}, \\
& =(-1)^{n-1} \varepsilon & & \text { if } x<x_{1} .
\end{aligned}
$$

Proof. The first assertion is rather obvious. In the second case, if $i<j$ then $g_{i}-g_{j}$ has the $n-1$ zeroes

$$
x_{1}, \ldots, x_{i-1} ; x_{i+1}, \ldots, x_{j-1} ; x_{j+1}, \ldots, x_{n+1}
$$

at which it must change sign, by Lemma 3.2. Further, $g_{i}(x)-g_{j}(x)$ is equal to $+D_{j}$ at $x=x_{j}$ and equal to $-D_{i}$ at $x=x_{i}$. These remarks easily imply (3.7) and (3.8). The following result is an immediate corollary.

(3.9) Lemma. Let $T$ be any subset of $(a, b)$ and let $f$ be a function on $T$ which is convex in the sense that

$$
L\left(x_{1}, \ldots, x_{n} ; f \mid x\right) \leqq f(x),
$$

whenever $x_{1} \leqq \cdots \leqq x_{n} \leqq x$ are all in $T$. Then

$$
(-1)^{n-i}\left[f(x)-L\left(x_{1}, \ldots, x_{n} ; f \mid x\right)\right] \geqq 0
$$

as soon as $x_{1}<\cdots<x_{n}$ and $x$ are all in $T$. Here, $i$ is chosen such that $x_{i} \leqq x \leqq x_{i+1}$, $\left(0 \leqq i \leqq n ; x_{0}=a, x_{n+1}=b\right)$.

The following Lemma extends a result of Moldovan [10, p. 53]. A certain special case due to Popoviciu [17, p. 56] was used by Ciesielski [3] in proving Theorem 2.2.

(3.12) Lemma. Let $x_{1}<x_{2}<\cdots<x_{N}$ be a fixed set of $N>n$ points in $(a, b)$. Let $f$ be any function on $(a, b)$ such that

$$
L\left(x_{i}, x_{i+1}, \ldots, x_{i+n-1} ; f \mid x_{i+n}\right) \leqq f\left(x_{i+n}\right)
$$

for all $i=1,2, \ldots, N-n$. Then

$$
L\left(x_{i_{1}}, x_{i_{2}}, \ldots, x_{i_{n}} ; f \mid x_{m}\right) \leqq f\left(x_{m}\right),
$$

whenever $1 \leqq i_{1}<i_{2}<\cdots<i_{n} \leqq m \leqq N$. 
Proof. Since (3.14) is obvious for $m=i_{n}$, we may assume that $i_{n}<m$. The proof goes by induction with respect to $M=m-i_{i}$, where $M \geqq n$. By (3.13), the assertion does hold for $M=n$; thus, let $M \geqq n+1$. There exists at least one index $i_{1}<s<m$ with $s \notin\left\{i_{1}, i_{2}, \ldots, i_{n} ; m\right\}$. Let the $n+1$ numbers $x_{i_{1}}, \ldots, x_{i_{n}}$ and $x_{s}$ be reordered as $x_{r_{1}}<x_{r_{2}}<\cdots<x_{r_{n+1}}$. Thus, $r_{1}=i_{1}$ and $r_{n+1} \leqq m-1=r_{1}+M-1$. By induction,

and

$$
L\left(x_{r_{2}}, \ldots, x_{r_{n+1}} ; f \mid x_{m}\right) \leqq f\left(x_{m}\right)
$$

$$
L\left(x_{r_{1}}, \ldots, x_{r_{n}} ; f \mid x_{r_{n+1}}\right) \leqq f\left(x_{r_{n+1}}\right) .
$$

In the notation of Lemma 3.6, replacing there $\left\{x_{1}, \ldots, x_{n+1}\right\}$ by $\left\{x_{r_{1}}, \ldots, x_{r_{n+1}}\right\}$, we are given that $g_{1}\left(x_{m}\right) \leqq f\left(x_{m}\right)$ and that $D_{n+1} \geqq 0$; here, $x_{m}>x_{r_{n+1}}$. It must be shown that $g_{j}\left(x_{m}\right) \leqq f\left(x_{m}\right)$ where $2 \leqq j \leqq n+1$ is chosen such that $r_{j}=s$.

One may as well assume that $g_{j} \not \equiv g_{1}$ in which case $D_{n+1}>0$ and $\varepsilon=1$. Applying (3.8) with $i=1$ and $x=x_{m}$, one obtains that

$$
g_{j}\left(x_{m}\right)<g_{1}\left(x_{m}\right) \leqq f\left(x_{m}\right),
$$

which is the desired result.

(3.15) Lemma. Let $E$ and $F$ be measurable subsets of $R$ such that $\mu(E)<\infty$, where $\mu$ denotes Lebesgue measure. Then both

$$
\phi(r)=\mu(r E \cap F) \quad \text { and } \quad \psi(r)=\mu((r+E) \cap F)
$$

are continuous functions of $r \in R$.

Proof. The proof that $\phi$ is continuous is analogous to the proof in [5, p. 266], of the well-known fact that $\psi$ is continuous. Letting $\rho(A, B)=\mu(A \Delta B)$, one has

$$
|\mu(c r E \cap F)-\mu(r E \cap F)| \leqq \rho(c r E \cap F, r E \cap F) \leqq|r| \rho(c E, E) .
$$

Using the regularity of $\mu$, it follows that the latter right-hand side tends to 0 as $c$ tends to 1 .

(3.16) LemMA. Let $B$ denote a measurable subset of $R$ of positive measure, and let $\left\{r_{j}\right\}$ be a dense sequence in $R$. Then, for almost all $h \in R$, we have $r_{j} h \in B$ for at least one index $j$ and $r_{k}+h \in B$ for at least one index $k$.

Proof. Let $M>0$ be arbitrary, and let $E$ denote the measurable set consisting of all numbers $h$ such that $|h|<M$ and $r_{j} h \notin B$ for each index $j$. Then the function $\phi(r)=\mu(r E \cap B)$ satisfies $\phi\left(r_{j}\right)=0$ for all $j$; hence, by Lemma 3.15, $\phi(r)=0$ for all real numbers $r$; therefore $\mu(E)=0$. For, otherwise, take $r$ such that $r e_{0}=b_{0}$ with $e_{0} \neq 0$ and $b_{0}$ as density points of $E$ and $B$ respectively. The proof of the second assertion is completely analogous.

4. Proof of Theorem 2.7. Let the function $f$ on $(a, b)$ be weakly $\mathscr{F}_{n}$-convex, that is, (2.3) holds whenever $a<x_{0}<x_{0}+n h<b$. Applying Lemma 3.12 with $x_{i}=x_{0}+i(h / q), i=0,1, \ldots, N$, it follows that

$$
f(x) \geqq L\left(x_{1}, \ldots, x_{n} ; f \mid x\right)
$$


whenever the numbers $x_{1}<\cdots<x_{n} \leqq x$ in $(a, b)$ are rationally comparable in the sense that, for a suitable number $h \neq 0$,

$$
x_{i}=x-r_{i} h \text { with } r_{i} \in Q, \quad(i=1, \ldots, n) .
$$

Here, $Q$ stands for the field of rational numbers.

Next, applying Lemma 3.9, we have for each $i=0,1, \ldots, n$ that

$$
(-1)^{n+1-i} f(x) \leqq(-1)^{n+1-i} L\left(x_{1}, \ldots, x_{n} ; f \mid x\right)
$$

whenever $x_{1}<\cdots<x_{n}$ and $x$ are rationally comparable and, further, $x_{i} \leqq x \leqq x_{i+1}$; $\left(x_{0}=a\right.$ and $\left.x_{n+1}=b\right)$.

(4.3) Now assume also that $f$ is bounded on some measurable set of positive measure. Let $E$ denote the (nonempty and closed) set of points $x \in(a, b)$ such that each neighborhood of $x$ contains a measurable subset of positive measure on which $f$ is bounded. Let further

$$
a^{\prime}=\inf \{x: x \in E\}, \quad b^{\prime}=\sup \{x: x \in E\}, \quad\left(a \leqq a^{\prime}<b^{\prime} \leqq b\right) .
$$

Note that both $a^{\prime}$ and $b^{\prime}$ are accumulation points of $E$.

(4.4) We assert that in the interval $\left(a^{\prime}, b\right)$ the function $f$ is locally bounded below. Let $a^{\prime}<x_{0}<b$ be given and choose points $z_{i} \in E$ such that $a^{\prime}<z_{1}<\cdots<z_{n}<x_{0}$. Choose $\delta>0$ such that $z_{i}>z_{i-1}+3 \delta,\left(i=1, \ldots, n+1 ; z_{0}=a, z_{n+1}=x_{0}\right)$. Next, choose $A_{i} \subset\left(z_{i}-\delta, z_{i}+\delta\right)$ as a measurable set of positive measure on which $f$ is bounded, $(i=1, \ldots, n)$. Observe that, for $x_{i} \in A_{i}(i=1, \ldots, n)$, we always have $a+2 \delta<x_{1}<x_{2}<\cdots<x_{n}<x_{0}$ and $x_{i+1}-x_{i}>\delta(i=1, \ldots, n-1)$. It follows from Theorem 3.1 that, for $x_{i}$ restricted to $A_{i}$ (so that $f\left(x_{i}\right)=y_{i}$ is bounded) and for $x$ restricted to a compact subinterval of $(a, b)$, the function $L\left(x_{1}, \ldots, x_{n} ; f \mid x\right)$ is uniformly bounded in $x_{1}, \ldots, x_{n}$ and $x$.

In view of (4.1) and these remarks, we see that in proving that $f$ is locally bounded below at $x_{0}$ it suffices to prove the following. Given a number $x$ (in some neighborhood of $x_{0}$ ) there always exists a number $h \neq 0$ such that, for each $i=1, \ldots, n$, we have $x-r_{i} h \in A_{i}$ for at least one rational number $r_{i}$. In fact, almost all numbers $h$ have this property as follows from Lemma 3.16 applied with $B=x-A_{i}$.

(4.5) We further assert that $f$ is locally bounded above in the interval $\left(a^{\prime}, b^{\prime}\right)$, (hence, it is locally bounded there). The proof is completely analogous to the proof of (4.4), choosing $z_{i} \in E(i=1, \ldots, n)$ such that

$$
a^{\prime}<z_{1}<\cdots<z_{n-1}<x_{0}<z_{n}<b^{\prime} .
$$

One further applies (4.2) with $i=n-1$.

(4.6) Let $a^{\prime}<x_{0}<b$. We assert that

$$
\liminf _{x \downarrow x_{0}} f(x) \geqq f\left(x_{0}\right) ; \quad \lim _{x \uparrow x_{0}} \sup _{x} f(x) \leqq f\left(x_{0}\right) .
$$

First, choose the closed intervals $A_{1}, \ldots, A_{n-1}$ such that

$$
a^{\prime}<A_{1}<\cdots<A_{n-1}<\min \left(x_{0}, b^{\prime}\right),
$$


(in an obvious notation). By (4.5), $f$ is bounded on each interval $A_{i}$. Using Theorem 3.1 , we see that for each $\varepsilon>0$ there exists a number $\delta>0$, depending only on $\varepsilon$ and $x_{0}$, such that

$$
\left|L\left(x_{1}, \ldots, x_{n-1}, x_{0} ; f \mid x\right)-f\left(x_{0}\right)\right|<\varepsilon
$$

as soon as $x_{i} \in A_{i}(i=1, \ldots, n-1)$ and $\left|x-x_{0}\right|<\delta$. Further, since $A_{i}$ is an interval, we have for each number $h=x-x_{0} \neq 0$ that $x_{i}=x_{0}-r_{i} h$ is in $A_{i}$ for some rational number $r_{i},(i=1, \ldots, n-1)$. We are now in a position to apply (4.2) (with $i=n$ if $x>x_{0}$, with $i=n-1$ if $x<x_{0}$ ) to the rationally comparable points $x_{1}<\cdots<x_{n-1}$ $<x_{n}=x_{0}$ and $x=x_{0}+h$. Using (4.8), one immediately obtains (4.7).

(4.9) Next we assert that, for $a^{\prime}<x_{0}<b^{\prime}$, we have

$$
\limsup _{x \downarrow x_{0}} f(x) \leqq f\left(x_{0}\right) ; \quad \liminf _{x \uparrow x_{0}} f(x) \geqq f\left(x_{0}\right) .
$$

The proof of (4.10) is completely analogous to the proof of (4.7). One chooses closed intervals $A_{1}, \ldots, A_{n-2}$ and $A_{n}$ such that $a^{\prime}<A_{1}<\cdots<A_{n-1}<x_{0}<A_{n}$. Afterwards, one applies (4.2), with $i=n-2$ or $i=n-1$, to a set of rationally comparable points $x=x_{0}+h, x_{i}=x_{0}-r_{i} h \in A_{i}$ when $i \neq n-1, x_{n-1}=x_{0}$.

(4.11) End of proof. By the second inequality (4.7), $f$ is bounded above in some left neighborhood of $x_{0}$ as soon as $a^{\prime}<x_{0}<b$. By (4.4), $f$ is also bounded below there. This proves that $b^{\prime}=b$. In a similar way, since $\tilde{f}(x)=(-1)^{n} f(-x)$ is convex with respect to the $n$-parameter family $\tilde{\mathscr{F}}_{n}=\left\{\tilde{g}: g \in \mathscr{F}_{n}\right\}$ in the interval $(-b,-a)$, we also have $\tilde{b}^{\prime}=\tilde{b}$, that is, $a^{\prime}=a$. We can now conclude from (4.7) and (4.10) that $f$ is continuous in the entire interval $\left(a^{\prime}, b^{\prime}\right)=(a, b)$.

(4.12) REMARK. In Theorem 2.7 and in its proof one could replace the property of $A$ being "measurable and of positive measure" by the property of $A$ being "a nonempty open set less some set of first category". Thus, it would be sufficient for the continuity of the weakly $\mathscr{F}_{n}$-convex function $f$ that $f$ be bounded on a Borel measurable set of second category, compare [8, p. 211]. An analogous modification of Theorem 2.2 was also indicated by Ciesielski [3].

5. Proof of Theorem 2.9. Let $f$ be an arbitrary weakly $\mathscr{F}_{n}$-convex function on $(a, b)$; no further assumptions will be made. Let $x_{0}$ and $h_{1}, \ldots, h_{k}$ be given numbers. Without loss of generality we may take the $h_{j}$ as being positive.

Let $Q$ denote the field of rational numbers, so that $Q^{k}$ is the collection of all $k$-tuples $r=\left(r_{1}, \ldots, r_{k}\right)$ of rational numbers. Define

$$
g(r)=f\left(x_{0}+r_{1} h_{1}+\cdots+r_{k} h_{k}\right)
$$

when $r \in E \cap Q^{k}$. Here, $E$ denotes the open strip in $R^{k}$ consisting of all $k$-tuples $r=\left(r_{1}, \ldots, r_{k}\right)$ of real numbers such that $a<x_{0}+\sum_{j=1}^{k} r_{j} h_{j}<b$. We must prove that $g$ can be uniquely extended to a continuous function on all of $E$. Since the domain $E \cap Q^{k}$ of $g$ is dense in $E$, it suffices to prove that $g$ is uniformly continuous on 
$K \cap Q^{k}$ whenever $K$ is a compact subset of $E$. In fact, it suffices that for any $\nu=1, \ldots, k$, for any subset $K$ of $E$ of the form

$$
K=\left\{r=\left(r_{1}, \ldots, r_{k}\right): \alpha_{j} \leqq r_{j} \leqq \beta_{j}(j=1, \ldots, k)\right\},
$$

and for any number $\varepsilon>0$, there exists a constant $\delta>0$ (depending on $\nu, K$, and $\varepsilon$ ), such that

$$
\left|g\left(r+\lambda_{\nu} \varepsilon_{v}\right)-g(r)\right|<\varepsilon,
$$

whenever both $r$ and $r+\lambda_{\nu} \varepsilon_{v}$ are in $K \cap Q^{k}$ and $\left|\lambda_{v}\right|<\delta$. Here, $\varepsilon_{v} \in Q^{k}$ has components $\delta_{v}^{j}(j=1, \ldots, k)$, while $\lambda_{v}$ is a scalar, $\lambda_{v} \in Q$.

(5.4) We shall first establish that $g$ (as defined by (5.1)) is locally bounded on its domain $E \cap Q^{k}$. It suffices to prove that $g$ is bounded on $K \cap Q^{k}$ whenever $K$ is a subset of $E$ of the form (5.2). The proof goes by induction with respect to $k$. Thus, let $k \geqq 1$ and suppose that assertion (5.4) is true when $k$ is replaced by a smaller positive integer.

Let $\rho_{i}(i=1, \ldots, n)$ be fixed rational numbers such that $\alpha_{k} \leqq \rho_{1}<\rho_{2}<\cdots<\rho_{n} \leqq \beta_{k}$. By induction, (replacing $x_{0}$ by $x_{0}+\rho_{i} h_{k}$ ), the quantity

$$
y_{i}=g\left(r_{1}, \ldots, r_{k-1}, \rho_{i}\right)
$$

is bounded as long as $\left(r_{1}, \ldots, r_{k-1}, \rho_{i}\right) \in K \cap Q^{k}$.

Next, we apply (4.2) with $x_{1}, \ldots, x_{n}$ and $x$ replaced by

$$
x_{i}=x_{0}+\sum_{j=1}^{k-1} r_{j} h_{j}+\rho_{i} h_{k}, \quad(i=1, \ldots, n)
$$

and

$$
x=x_{0}+\sum_{j=1}^{k-1} r_{j} h_{j}+r_{k} h_{k}
$$

respectively. Here $r_{1}, \ldots, r_{k}$ are taken as rational numbers such that

$$
r=\left(r_{1}, \ldots, r_{k}\right) \in K \text {. }
$$

Observe that $x_{1}<x_{2}<\cdots<x_{n}$ and that the $n+1$ numbers $x_{1}, \ldots, x_{n}$ and $x$ are rationally comparable. In this way, (4.2) and (5.1) yield that

$$
(-1)^{n+1-i} g(r) \leqq(-1)^{n+1-i} L\left(x_{1}, \ldots, x_{n} ; y_{1}, \ldots, y_{n} \mid x\right)
$$

whenever $r \in K \cap Q^{k}$. Here, the integer $i$ is to be chosen such that $0 \leqq i \leqq n$ and $\rho_{i} \leqq r_{k} \leqq \rho_{i+1},\left(\rho_{0}=\alpha_{k}\right.$ and $\left.\rho_{n+1}=\beta_{k}\right)$.

As to the right-hand side of (5.6), the variables $x$ and $x_{i}$ are restricted to a compact subinterval of $(a, b)$ while the quantities $y_{i}$ (as defined by (5.5)) are uniformly bounded. Moreover, $\left|x_{i+1}-x_{i}\right|=\left|\rho_{i+1}-\rho_{i}\right| h_{k}>\delta(i=1, \ldots, k)$ for some positive contant $\delta$. It follows from Theorem 3.1 that the right-hand side of (5.6) is uniformly bounded for each fixed choice of the set of rational numbers $\left\{\rho_{1}, \ldots, \rho_{n}\right\}$ in $\left[\alpha_{k}, \beta_{k}\right]$. Varying this set, one easily obtains from (5.6) that $g$ is bounded on $K \cap Q^{k}$. 
(5.7) In establishing the assertion (5.3) we shall take $\nu=k$ for convenience. Let $K$ be the compact subinterval of $E$ defined by (5.2). Let $\pi$ denote the projection

$$
\pi(r)=x_{0}+\sum_{j=1}^{k} r_{j} h_{j}
$$

of $E$ onto $(a, b)$. Let $\left[a^{\prime}, b^{\prime}\right]$ denote the $\pi$-projection of $K, a<a^{\prime}<b^{\prime}<b$. Let $1 \leqq i \leqq n$ be a fixed integer and choose numbers $z_{1}, \ldots, z_{i-1}, z_{i+1}, \ldots, z_{n}$ such that

$$
a<z_{1}<\cdots<z_{i-1}<a^{\prime}<b^{\prime}<z_{i+1}<\cdots<z_{n}<b .
$$

Take $\eta>0$ so small that the mutual distances between the latter $n+3$ numbers are all $\geqq 3 \eta$.

For each point $r \in K \cap Q^{k}$, choose $\lambda_{j}=\lambda_{j}(r) \in Q$ in some fixed manner such that

$$
\left|\pi(r)+\lambda_{j} h_{k}-z_{j}\right|<\eta, \quad(j=1, \ldots, n ; j \neq i) .
$$

Clearly, each $\lambda_{j}(r)$ is bounded.

Consider a point $r \in K \cap Q^{k}$ and define $x_{i}=x_{i}(r)=\pi(r)$. For $j=1, \ldots, n, j \neq i$, define $x_{j}=\pi(r)+\lambda_{j}(r) h_{k}$. We want to apply (4.2) with these $x_{j}$ and with $x=\pi(r)$ $+\lambda h_{k}(\lambda \in Q)$ so as to prove (5.3) with $\nu=k$.

Observe that $a^{\prime} \leqq x_{i} \leqq b^{\prime}$, while $\left|x_{j}-z_{j}\right|<\eta$ when $j \neq i$. Consequently, we have

$$
a<x_{1}<\cdots<x_{i-1}<a^{\prime}<x_{i}<b^{\prime}<x_{i+1}<\cdots<x_{n}<b,
$$

in such a way that $\left|x_{j+1}-x_{j}\right|>\eta$ for $j=0,1, \ldots, n ;\left(x_{0}=a\right.$ and $\left.x_{n+1}=b\right)$. It follows from assertion (5.4) that the quantity

$$
y_{j}=f\left(x_{j}(r)\right)=g\left(r+\lambda_{j}(r) \varepsilon_{k}\right),
$$

$\left(j=1, \ldots, n ; \lambda_{i}(r)=0\right)$ is a bounded function of $r \in K \cap Q^{k}$. Here, we did use the fact that $\lambda_{j}(r)$ itself is bounded, that $K$ is compact, and that $a+\eta<x_{j}(r)<b-\eta$ for all $j$.

It follows from these remarks, together with Theorem 3.1 , that the continuity in $x$ of the function

$$
L_{r}(x)=L\left(x_{1}(r), \ldots, x_{n}(r) ; f \mid x\right)
$$

is uniform in both $x$ and $r$ as long as $a^{\prime} \leqq x \leqq b^{\prime}$ and $r \in K \cap Q^{k}$. Hence, letting $L_{r}^{*}(s)=L_{r}(\pi(s))$, we have that the continuity in $s$ of the function $L_{r}^{*}(s)$ on $E$ is uniform in both $r$ and $s$ as long as $r \in K \cap Q^{k}$ and $s \in K$. Observing that

$$
L_{r}^{*}(r)=L_{r}\left(x_{i}(r)\right)=y_{i}=g(r),
$$

we conclude that, for each number $\varepsilon>0$, there exists a number $\delta>0$ (depending only on $\varepsilon$ and $K$ ) such that

$$
\left|L_{r}^{*}(s)-g(r)\right|<\varepsilon \text { when } r, s \in K \cap Q^{k} \quad \text { and } \quad|r-s|<\delta .
$$

We are now in a position to use (4.2) with $x_{1}=x_{1}(r), \ldots, x_{n}=x_{n}(r)$ as above and with

$$
x=\pi\left(r+\lambda \varepsilon_{k}\right)=x_{i}(r)+\lambda h_{k} .
$$


In particular, $x_{1}, \ldots, x_{n}$ and $x$ are rationally comparable. Moreover, as long as $r+\lambda \varepsilon_{k} \in K$ we have either $x_{i} \leqq x<x_{i+1}$ or $x_{i-1}<x \leqq x_{i}$ depending on $\gamma=\operatorname{sgn}(\lambda)$. We conclude from (4.2) that

$$
\begin{aligned}
\sigma g\left(r+\lambda \varepsilon_{k}\right) & =\sigma f\left(x_{i}(r)+\lambda h_{k}\right) \leqq \sigma L_{r}\left(x_{i}(r)+\lambda h_{k}\right) \\
& =\sigma L_{r}^{*}\left(r+\lambda \varepsilon_{k}\right) \leqq \sigma g(r)+\varepsilon,
\end{aligned}
$$

provided both $r$ and $r+\lambda \varepsilon_{k}$ are in $K \cap Q^{k}$, and further $\lambda$ is a rational number satisfying $|\lambda|<\delta$. Here, we used (5.10). Further, $\sigma$ is defined by $\sigma=(-1)^{n+1-i+\gamma}$ with $\gamma=\operatorname{sgn}(\lambda)$. Since $i$ can be both even and odd, (5.11) immediately yields the desired uniform continuity (5.3).

(5.12) Remark. Observe that in the above proof we did not use (2.3) in its full strength, but only for values $h>0$ of the form $h=r h_{j}$ for some $r \in Q$ and some $j=1, \ldots, k$.

Theorem 2.9 and its proof would remain valid if throughout the ring $Q$ of rational numbers is replaced by the ring $Q^{\prime}$ of rational numbers of the form $m \cdot 2^{-k}$ with $m$ and $k$ as integers. Accordingly, (2.3) would only be needed for $h=2^{-k}(k=1$, $2, \ldots)$.

6. Strongly convex functions. Let $\mathscr{F}_{n}$ be a given $n$-parameter family on $(a, b)$. A function $f$ on $(a, b)$ is said to be $\mathscr{F}_{n}$-convex (or strongly $\mathscr{F}_{n}$-convex) when (2.12) holds for each choice of the numbers $x_{1}<\cdots<x_{n+1}$ in $(a, b)$. If so then, by Lemma 3.9 , we even have that

$$
(-1)^{n+1-i} f(x) \leqq(-1)^{n+1-i} L\left(x_{1}, \ldots, x_{n} ; f \mid x\right),
$$

whenever $a<x_{1}<\cdots<x_{n}<b$. Here, $0 \leqq i \leqq n$ is chosen such that $x_{i} \leqq x \leqq x_{i+1}$, $\left(x_{0}=a\right.$ and $\left.x_{n+1}=b\right)$.

As was observed by Moldovan [10, p. 63], an $\mathscr{F}_{n}$-convex function $f$ is always continuous; (to prove continuity at $x_{0}$ one simply applies (6.1) and Theorem 3.1 with $x_{n}=x_{0}$ or $x_{n-1}=x_{0}$ ). The converse part of Lemma 2.13 can be seen as follows.

Let the function $f$ on $(a, b)$ be continuous and weakly $\mathscr{F}_{n}$-convex. By Theorem 3.1, the left-hand side of (2.12) is a jointly continuous function of $x_{1}, \ldots, x_{n}$ and $x_{n+1}$ as long as $x_{1}<\cdots<x_{n}$. By (4.1), we have (2.12) as soon as the numbers $x_{1}<\cdots<x_{n+1}$ are rationally comparable, hence, also without this restriction; that is, $f$ is (strongly) $\mathscr{F}_{n}$-convex.

The main purpose of the present section is to prove Theorem 2.30. In the proof we shall make essential use of the following Theorem 6.2, which is a special case of a result of Butzer and Kozakiewicz [2]. Here, $\nabla_{h}$ denotes the operator defined by

$$
\left(\nabla_{h} g\right)(x)=[g(x+h)-g(x-h)] /(2 h) .
$$

(6.2) THEOREM. Let $k$ be a fixed positive integer and let $f$ be a function defined on the open subinterval $J$ of $R$ such that $\nabla_{h}^{k} f(x)$ is uniformly bounded as long as $x$ is 
restricted to a compact subinterval of $J$ and $h$ is sufficiently small. Suppose further that the following limit exists almost everywhere in $J$.

$$
\lim _{h \downarrow 0} \nabla_{h}^{k} f(x)=\phi(x) .
$$

Then $f$ admits throughout $J a(k-1)$ th derivative and, moreover, this derivative is given by

$$
f^{(k-1)}(x)=f^{(k-1)}\left(x_{0}\right)+\int_{x_{0}}^{x} \phi(t) d t, \quad \text { for all } x \in J .
$$

In the sequel, $f$ will denote a fixed $\mathscr{F}_{n}$-convex function on $(a, b)$. Further, $\Omega$ will denote the collection of all ordered $n$-tuples $X=\left(x_{1}, \ldots, x_{n}\right)$ consisting of $n$ distinct numbers in $(a, b), x_{1}<x_{2}<\cdots<x_{n}$. The $i$ th element $x_{i}$ of $X$ will also be denoted as $x_{i}(X)$. Let further

$$
L(X \mid x)=L\left(x_{1}, \ldots, x_{n} ; f \mid x\right) \quad \text { for } X \in \Omega, x \in(a, b) .
$$

More precisely, $L(X \mid \cdot)$ denotes the unique member $g \in \mathscr{F}_{n}$ such that for the points $x_{i}=x_{i}(X)$ one has $f\left(x_{i}\right)=g\left(x_{i}\right), i=1, \ldots, n$.

Let $z_{1}<\cdots<z_{n}$ be fixed numbers in $(a, b)$. We shall repeatedly make use of the obvious relation

$$
L(X \mid y)=L\left(z_{1}, \ldots, z_{n} ; y_{1}, \ldots, y_{n} \mid y\right),
$$

where $y_{i}=L\left(X \mid z_{i}\right), i=1, \ldots, n$. Observe that, by Theorem 3.1, the right-hand side of (6.6) is a continuous function of the $n+1$ variables $y_{1}, \ldots, y_{n}$ and $y$. Similarly, since $f$ is continuous, the function $L(X \mid x)$ on $\Omega \times(a, b)$ is jointly continuous in $X$ and $x$ when $\Omega$ is given the natural topology it inherits when regarded as a subset of $(a, b)^{n}$.

In $\Omega$ we shall further employ the partial ordering defined by

$$
X^{\prime} \leqq X^{\prime \prime} \quad \text { iff } \quad x_{i}\left(X^{\prime}\right) \leqq x_{i}\left(X^{\prime \prime}\right) \quad \text { for } i=1, \ldots, n .
$$

We write $X \leqq x$ if $x_{i}(X) \leqq x$ for all $i$. We write $X<x$ if $x_{i}(X)<x$ for all $i$. We write $X \rightarrow x$ if each $x_{i}(X)$ tends to $x$, and so on. The following lemma is a crucial step in the proof.

(6.8) Lemma. Let $z \in(a, b)$ and $X^{\prime}, X^{\prime \prime} \in \Omega$. Then

$$
L\left(X^{\prime} \mid z\right) \leqq L\left(X^{\prime \prime} \mid z\right) \leqq f(z) \quad \text { whenever } X^{\prime} \leqq X^{\prime \prime}<z .
$$

Proof. The second inequality follows from (6.1) with $i=n$. Next, let $X^{\prime} \leqq X^{\prime \prime}<z$. Put $x_{i}\left(X^{\prime}\right)=x_{i}^{\prime}$ and $x_{i}\left(X^{\prime \prime}\right)=x_{i}^{\prime \prime}$; hence $x_{i}^{\prime} \leqq x_{i}^{\prime \prime},(i=1, \ldots, n)$. Consider further the n-tuples

$$
X_{i}=\left\{x_{1}^{\prime}, \ldots, x_{i}^{\prime}, x_{i+1}^{\prime \prime}, \ldots, x_{n}^{\prime \prime}\right\} \in \Omega, \quad(i=0,1, \ldots, n) .
$$

Clearly, $X^{\prime}=X_{n} \leqq X_{n-1} \leqq \cdots \leqq X_{0}=X^{\prime \prime}$; thus it suffices to prove that $L\left(X_{i} \mid z\right)$ $\leqq L\left(X_{i-1} \mid z\right)$ for $i=1, \ldots, n$. Let $i$ be fixed. 
In the notation of Lemma 3.6, replacing

$$
\left(x_{1}^{\prime}, \ldots, x_{i-1}^{\prime}, x_{i}^{\prime}, x_{i}^{\prime \prime}, x_{i+1}^{\prime \prime}, \ldots, x_{n}^{\prime \prime}\right\} \quad \text { by }\left\{x_{1}, \ldots, x_{n+1}\right\} \text {, }
$$

we know that $D_{n+1} \geqq 0$ (by (6.1) with $i=n$ ) and we must prove that $g_{i+1}(z) \leqq g_{i}(z)$, where $z>x_{n+1}$. But this is an immediate consequence of Lemma 3.6, in particular of (3.8) if $D_{n+1}>0$.

(6.10) The following result holds for every $n$-parameter family $\mathscr{F}_{n}$. Though we shall not use it in the proof of Theorem 2.30, it does stress the central idea behind the proof. Moreover, it has an independent interest of its own. One can prove analogous results for the case that $X=\left(x_{1}, \ldots, x_{n}\right)$ does not converge to a single point but to an $n$-tuple $X^{0}=\left(x_{1}^{0}, \ldots, x_{n}^{0}\right)$ having one or several groups of equal elements $x_{i}^{0}$.

Recall that $L(X \mid x)$ is defined by (6.5), where $f$ is a fixed $\mathscr{F}_{n}$-convex function on $(a, b)$.

(6.11) THEOREM. To each $x \in(a, b)$ there correspond unique members $g_{\bar{x}}^{-}(\cdot)$ and $g_{x}^{+}(\cdot)$ of $\mathscr{F}_{n}$, such that

$$
\lim _{x \uparrow x} L(X \mid y)=g_{x}^{-}(y) \quad \text { and } \quad \lim _{x \downarrow x} L(X \mid y)=g_{x}^{+}(y)
$$

holds for each $y \in(a, b)$. Moreover, there exists a finite or denumerable set $D$ in $(a, b)$ such that

$$
\lim _{X \rightarrow x} L(X \mid y)=g_{x}^{+}(y) \quad \text { for each } x \notin D \text { and each } y,
$$

$(x, y \in(a, b))$. In particular, $g_{x}^{-}=g_{x}^{+}$for each $x \notin D$.

(6.14) For the moment, let $a<z<b$ be fixed and restrict $x$ to the interval $a<x<z$. Consider the functions

$$
\phi_{z}^{-}(x)=\sup \{L(X \mid z): X \in \Omega, X \leqq x\}
$$

and

$$
\phi_{z}^{+}(x)=\inf \{L(X \mid z): X \in \Omega, x \leqq X<z\} .
$$

Each of these functions is obviously nondecreasing so that $\phi_{z}^{-}(x+0)$ and $\phi_{z}^{+}(x-0)$ exist. Since $L(X \mid z)$ is a continuous function of $X$, we have that $\phi_{z}^{-}(x)=\phi_{z}^{-}(x-0)$ is continuous to the left, similarly, $\phi_{z}^{+}(x)=\phi_{z}^{+}(x+0)$. It follows from Lemma 6.8 that

$$
\lim _{X \uparrow x} L(X \mid z)=\phi_{z}^{-}(x) \quad \text { and } \quad \lim _{X \downarrow x} L(X \mid z)=\phi_{z}^{+}(x) .
$$

It also follows from Lemma 6.8 that

$$
\phi_{z}^{-}(x) \leqq \phi_{z}^{+}(x) \leqq f(z) \quad \text { and } \quad \phi_{z}^{+}(x) \leqq \phi_{z}^{-}(y) \quad \text { when } x<y,
$$

(choose $X \in \Omega$ such that $x<X<y$ ). Letting $y \downarrow x$ or $x \uparrow y$ these in turn yield that

$$
\phi_{z}^{+}(x-0) \leqq \phi_{z}^{-}(x) \leqq \phi_{z}^{+}(x) \leqq \phi_{z}^{-}(x+0) .
$$


Consequently, the set

$$
D_{z}=\left\{x: a<x<z, \phi_{z}^{-}(x) \neq \phi_{z}^{+}(x)\right\}
$$

is finite or denumerable. In fact, $D_{z}$ is precisely the set of points $x \in(a, z)$ where the nondecreasing functions $\phi_{z}^{-}$and $\phi_{z}^{+}$make a jump. Moreover, $\phi_{z}^{-}(x)=\phi_{z}^{+}(x-0)$ everywhere; similarly, $\phi_{z}^{+}(x)=\phi_{z}^{-}(x+0)$. Finally, from Lemma 6.8, (6.15), (6.16), and (6.18), we conclude that

$$
\lim _{x \rightarrow x} L(X \mid z)=\phi_{z}^{+}(x) \quad \text { whenever } x \notin D_{z}, x<z .
$$

(6.20) Proof of Theorem 6.11. By (6.17), the limits (6.12) exist when $x<y$, in which case, $g_{x}^{-}(y)=\phi_{y}^{-}(x)$ and $g_{x}^{+}(y)=\phi_{y}^{+}(x)$. Applying (6.6), with $a<z_{1}<\cdots$ $<z_{n}<b$ fixed, and putting $D_{z_{1}} \cup \cdots \cup D_{z_{n}}=D$ one easily obtains all the stated assertions as long as $x<z_{1}$. But note that $z_{1}$ can be arbitrarily close to $b$.

(6.21) Proof of Theorem 2.30. We now assume that the $n$-parameter family $\mathscr{F}_{n}$ satisfies condition $C_{k}^{*}$ described in (2.28), with $k$ as an integer satisfying $1 \leqq k \leqq n-1$.

Let $a<z_{1}<\cdots<z_{n}<b$ be arbitrary but fixed. It follows from (2.29) and (6.6) that the derivative

$$
L^{(k)}(X \mid y)=\left(\frac{d}{d y}\right)^{k} L\left(x_{1}, \ldots, x_{n} ; f \mid y\right)
$$

(with $X=\left\{x_{1}, \ldots, x_{n}\right\}$ ) can be represented as a jointly continuous function $\Phi$ of $y$ and the $n$ quantities $y_{i}=L\left(X \mid z_{i}\right)$. That is,

$$
L^{(k)}(X \mid y)=\Phi\left(L\left(X \mid z_{1}\right), \ldots, L\left(X \mid z_{n}\right) ; y\right),
$$

for all $X \in \Omega$ and all $y \in(a, b)$. Here, $\Phi$ is a continuous function on $R^{n} \times(a, b)$.

Let $I$ be a fixed compact subinterval of $(a, b)$ and choose the above points $z_{i}$ to the right of $I$. As long as $X$ is in $I$, each quantity $L\left(X \mid z_{i}\right)$ is bounded; this follows from (6.9) with $X^{\prime}$ to the left of $I$ and $z=z_{1}$. Hence, by (6.22), there exists a constant $M$ (depending on $I$ only) such that

$$
\left|L^{(k)}(X \mid y)\right| \leqq M,
$$

as long as $X$ and $y$ are in $I$. As a further consequence, we have that the right-hand side of (6.22) is uniformly continuous jointly in terms of the $n+1$ quantities $L\left(X \mid z_{i}\right)$ and $y$, as long as $X$ and $y$ are in $I$. We now conclude, from (6.19) and (6.22), that

$$
\lim _{x \rightarrow x ; y \rightarrow x} L^{(k)}(X \mid y)=\phi(x) \quad \text { whenever } x \notin D, x<z_{1} .
$$

Here,

$$
\phi(x)=\Phi\left(\phi_{z_{1}}^{+}(x), \ldots, \phi_{z_{n}}^{+}(x) ; x\right),
$$

while $D=D_{z_{1}} \cup \cdots \cup D_{z_{n}}$ is a finite or denumerable set; (to begin with one obtains (6.24) only for points $x$ interior to $I$; but afterwards one can vary the interval $I$ ). 
From the known properties of the functions $\phi$ and $\phi_{z_{1}}^{+}$it follows that the function $\phi$ defined by (6.25) is locally bounded and has only discontinuities of the first kind in the interval $\left(a, z_{1}\right)$. Moreover, $\phi(x)$ is continuous at each point $x \notin D$ with $x<z_{1}$.

For the moment, let $x \in(a, b)$ and $h>0$ be fixed. Let $X_{h}=\left\{x_{1}, \ldots, x_{n}\right\}$ denote the $n$-tuple defined by

$$
x_{i}=x-k h+(i-1)(2 h), \quad(i=1, \ldots, n) .
$$

Since $L\left(X_{h} \mid x_{i}\right)=f\left(x_{i}\right)$ for $i=1, \ldots, n$ and $n \geqq k+1$, we have

$$
\begin{aligned}
\left(\nabla_{h}^{k} f\right)(x) & =(2 h)^{-k} \sum_{i=1}^{k+1}(-1)^{k+1-i}\left(\begin{array}{c}
k \\
i-1
\end{array}\right) f\left(x_{i}\right) \\
& =\nabla_{h}^{k} L\left(X_{h} \mid x\right)=L^{(k)}\left(X_{h} \mid y_{h}\right),
\end{aligned}
$$

for some number $y_{h}$ satisfying $\left|y_{h}-x\right|<k h$.

In this way we obtain from (6.23) that $\nabla_{h}^{k} f(x)$ is uniformly bounded as long as $x \in I$ and $h$ is sufficiently small. Moreover, (6.24) yields that

$$
\lim _{h \downarrow 0}\left(\nabla_{h}^{k} f\right)(x)=\phi(x) \quad \text { whenever } x \notin D, x<z_{1} .
$$

We now conclude from Theorem 6.2 that $f$ admits a $(k-1)$ th derivative of the form (6.4) throughout $\left(a, z_{1}\right)$. From the above properties of $\phi$, and since $z_{1}$ is arbitrary, this proves that $f$ belongs to the class $D_{k}$ described in (2.26).

(6.27) The above results (2.30), (6.8), and (6.11) concern the analytic properties of a function which is known to be convex relative to a given $n$-parameter family of functions. The referee kindly pointed out that there exist interesting parallels between these analytic results and certain geometric results due to J. Haller, O. Haupt, A. Kneser, N. Lane, P. Scherk and K. Singh, as reported in [23, p. 184; $198 \mathrm{ff}$; $230 ; 399 \mathrm{ff}$.]. Here, one is given an $n$-parameter family $\mathscr{F}_{n}$ of planar curves and further a curve $B$ with the property that each member of $\mathscr{F}_{n}$ intersects $B$ in at most $n$ points. One is interested, among other things, in establishing the existence or uniqueness of curves in $\mathscr{F}_{n}$ which are tangent curves or higher order osculating curves to $B$.

7. Miscellaneous results. We know from Theorem 2.2 that a function $f$ on $(a, b)$ is continuous if

$$
\Delta_{h}^{n} f(x) \geqq 0 \quad \text { for } a<x<b-n h
$$

holds for each $h>0$ and, moreover, $f$ is bounded on some measurable set of positive measure. By Theorem 2.7, the analogous result holds when (7.1) is replaced by

$$
L(x, x+h, \ldots, x+(n-1) h ; f \mid x+n h) \leqq f(x+n h),
$$

with $L$ as the interpolating function relative to a given $n$-parameter family $\mathscr{F}_{n}$ in $(a, b)$. 
The question arises in how far (7.1) or (7.2) can be weakened if $f$ is known to be measurable. The following is one result in this direction.

(7.3) THeOREM. Let $f$ be a given measurable function on $(a, b)$. Let $\left\{m_{k}\right\}$ be a given increasing sequence of integers and suppose that (7.2) holds for each $h=2^{-m_{k}}$ $(k=1,2, \ldots ; a<x<b-n h)$. Then there exists a (continuous) $\mathscr{F}_{n}$-convex function $g$ such that $f(x)=g(x)$ almost everywhere.

(7.4) Remark. Actually, by Lemma 3.12, (7.2) for all $h=2^{-m_{k}}$ is equivalent to (7.2) for all $h=2^{-k}$. It is easy to describe all the functions $f$ satisfying (7.2) for each $h=2^{-k}(k=1,2, \ldots)$. Namely, let $Q^{\prime}$ denote the set of all binary numbers $r=p \cdot 2^{-q}$ with $p$ and $q$ as integers. Within each coset $x+Q^{\prime}$, choose $f$ equal to a (continuous) $\mathscr{F}_{n}$-convex function $g$ (for instance, $g \in \mathscr{F}_{n}$ ), possibly using different functions $g$ in different cosets; see (5.12).

One can easily attain that the function $f$ thus constructed is locally bounded without being measurable, hence, in Theorem 7.3 the assumption that $f$ be measurable is essential. By choosing the same function $g$ for each coset $x+Q^{\prime}$ disjoint from a given null set, one sees that the assertion of Theorem 7.3 cannot be strengthened. On the other hand (by an obvious modification of the proof below) it would suffice to require the condition (7.2) of Theorem 7.3 only for almost all $a<x<b-n h$ (and each $h=2^{-m_{k}}$ ).

(7.5) Proof of Theorem 7.3. It suffices to prove the existence of a continuous function $g$ equal to $f$ almost everywhere. For, afterwards, $g$ is easily seen to be $\mathscr{F}_{n}$-convex, compare the proof of Lemma 2.13 at the beginning of $\S 6$.

Since $f$ is measurable, each subinterval of $(a, b)$ contains a measurable set $A$ of positive measure on which $f$ is bounded. By Lemma 3.16, we have for almost all $x$ that $x-r \in A$ for some $r \in Q^{\prime}$. The usual reasoning, as in (4.4) and (4.5), now yields the existence of a null set $N$ (that is, $\mu(N)=0$ ) such that $f$ is bounded on each set

$$
I_{\varepsilon}=\{x: a+\varepsilon \leqq x \leqq b-\varepsilon, x \notin N\}, \quad(\varepsilon>0) .
$$

One may assume that $N+Q^{\prime}=N$.

(7.6) Using the above boundedness, a standard reasoning (see (4.6) and (4.9)) yields that

$$
\lim _{r \rightarrow 0 ; r \in Q^{\prime}} f(x+r)=f(x) \quad \text { for each } x \notin N,
$$

the limit holding uniformly when $x$ is restricted to a set $I_{\varepsilon}(\varepsilon>0)$.

(7.7) Next, consider the functions

$$
f^{\prime}(x)=\text { ess } \liminf _{y \rightarrow x} f(y) ; \quad f^{\prime \prime}(x)=\text { ess } \lim _{y \rightarrow x} \sup f(y) .
$$

It is well known and easily shown that $f^{\prime}(x) \leqq f(x) \leqq f^{\prime \prime}(x)$ for almost all $x \in(a, b)$. Moreover, the function $f^{\prime}$ is lower semicontinuous while $f^{\prime \prime}$ is upper semicontinuous. In fact, these assertions would hold for an arbitrary function $f$, measurable or not. 
It suffices to prove that $f^{\prime}(x)=f^{\prime \prime}(x)$ for each $x \in(a, b)$. For, then $f^{\prime}=f^{\prime \prime}=g$ is a continuous function equal to $f$ almost everywhere.

Let $x_{0} \in(a, b)$ be fixed, and choose $\varepsilon>0$ such that $a+\varepsilon<x_{0}<b-\varepsilon$. Let $\eta>0$ be arbitrary but fixed and choose $\delta=\delta(\varepsilon, \eta)$ as a positive constant such that

$$
|f(x+r)-f(x)|<\eta \quad \text { whenever } x \in I_{\varepsilon}, r \in Q^{\prime},|r|<\delta .
$$

This is possible by (7.6).

Let $U$ be any neighborhood of $x_{0}$. Since $f$ is measurable there exists a measurable subset $A$ of $U$ of positive measure such that $f(x)>f^{\prime \prime}\left(x_{0}\right)-\eta$ throughout $A$. One may assume that $A \subset I_{\varepsilon}$ and further that all points of $A$ are density points of $A$.

Let $B$ denote the set of points $y$ such that $y=x+r$ for some $x \in A$ and some $r \in Q^{\prime}$ with $|r|<\delta$. It follows that

$$
f(y)>f^{\prime \prime}\left(x_{0}\right)-2 \eta \quad \text { for each } y \in B .
$$

Let $B^{\prime}$ denote the complement of $B$. By the definition of $B$, we have $\mu\left(B^{\prime} \cap(A+r)\right)$ $=0$ for each $r \in Q^{\prime}$ with $|r|<\delta$, hence (by Lemma 3.15), for each real number $r$ with $|\boldsymbol{r}| \leqq \delta$. It follows that each density point of $B^{\prime}$ is at a distance $\geqq \delta$ from each (density) point of $A$. Therefore, almost all points $y$, within a distance $\delta$ from the set $A$, are in $B$ and thus satisfy $f(y)>f^{\prime \prime}\left(x_{0}\right)-2 \eta$. Since $\delta>0$ is fixed and $A \subset U$ can be arbitrarily close to $x_{0}$, we conclude that $f^{\prime}\left(x_{0}\right)>f^{\prime \prime}\left(x_{0}\right)-2 \eta$. But $\eta>0$ is arbitrary, thus, $f^{\prime}\left(x_{0}\right)=f^{\prime \prime}\left(x_{0}\right)$.

(7.8) Let us now consider a somewhat different generalization of the relation

$$
\Delta_{h}^{n} f(x)=\sum_{j=0}^{n}(-1)^{n-j}\left(\begin{array}{l}
n \\
j
\end{array}\right) f(x+j h) \geqq 0
$$

namely, the relation

$$
\sum_{j=0}^{n} a_{j} f\left(x+T_{j} y\right) \geqq 0 \quad \text { for each } y \in B .
$$

Here, $T_{0}, T_{1}, \ldots, T_{n}$ denote given linear transformations in $p$-dimensional Euclidean space $R^{p}$. We shall assume that $T_{j}-T_{k}$ is nonsingular whenever $j \neq k$; for example, $T_{j} y=j \cdot y$ for each $y \in R^{p}$. Further, $a_{0}, a_{1}, \ldots, a_{n}$ denote given real numbers not all zero.

A (real-valued) function $f$ is said to satisfy (7.10) in an open subset $U$ of $R^{p}$ if it is defined on $U$ and, moreover, the inequality (7.10) holds for each pair $x \in R^{p}$, $y \in B$ such that $x+T_{j} y \in U,(j=0,1, \ldots, n)$.

Here, $B$ denotes a subset of $R^{p}$, usually close to the origin. It would in general be a serious loss of generality to take $B$ as a full neighborhood of 0 . For instance, if $n$ is odd and (7.9) had to hold for all small $h$ then it would have to hold with the equality sign for all $h$.

We shall henceforth also assume that

$$
a_{0}+a_{1}+\cdots+a_{n}=0
$$


so that the $a_{j}$ have both signs. Namely, (7.11) is at least a necessary condition in order that each bounded solution of $(7.10)$ be measurable or that each measurable solution be continuous. For example, if $\sum a_{j}=1$ (say) then, for any bounded function $g$ and any constant $c \geqq\left(\sum\left|a_{j}\right|\right)$ sup $|g|$, the function $f(x)=c+g(x)$ would satisfy (7.10), (irrespective of the particular structure of (7.10)). On the other hand, (7.11) is not quite sufficient for a measurable solution to be continuous, compare (7.18).

A measurable subset $B$ of $R^{p}$ will be said to be of positive upper density at 0 if there exists a positive constant $\varepsilon>0$ and a sequence $\left\{\delta_{i}\right\}$ of positive numbers with $\delta_{i} \downarrow 0$, such that, for all $i$,

$$
\left\{y \in B:|y|<\delta_{i}\right\} \geqq \varepsilon\left(\delta_{i}\right)^{p} .
$$

As an important example, take $B$ as a nonempty open cone with vertex 0 . The following theorem generalizes a result of Kurepa [9, p. 16].

(7.12) THEOREM. Let $f$ satisfy (7.10) in the open set $U \subset R^{p}$, with $B$ as a measurable subset of $R^{p}$ having a positive upper density at 0 . Suppose further that $f$ is bounded on a measurable subset $A$ of $U$ of positive measure. Then $f$ is bounded on some nonempty open set $V \subset U$ such that $V$ contains almost all points of $A$.

Moreover, if $f$ is also measurable on $U$ then it is locally bounded everywhere in $U$.

Proof. Put $f_{j}(x)=a_{j} f(x)(j=0,1, \ldots, n)$, thus,

$$
\sum_{j=0}^{n} f_{j}\left(x+T_{j} y\right) \geqq 0
$$

if $y \in B$ and $x+T_{j} y \in U(j=0,1, \ldots, n)$. Here, each function $f_{j}$ is bounded on $A$. Since $a_{j}$ can have both signs it suffices to show (as to the first assertion) that each $f_{j}$ is bounded below on an open set containing almost all points of $A$; (similarly for the second assertion). For definiteness, let us do this for the function $f_{0}$. Introducing $x^{\prime}=x+T_{0} y$, one may assume that $T_{0}=0$. One may also assume that $\mu(B)<\infty$.

For each $x \in U$, consider the points $x_{j}=T_{j}^{-1} x(j=1, \ldots, n)$ and the sets

$$
C_{x}=\bigcap_{j=1}^{n} T_{j}^{-1}(A-x)=\bigcap_{j=1}^{n}\left(T_{j}^{-1} A-x_{j}\right) ; \quad B_{x}=B \cap C_{x} .
$$

We claim that the set

$$
V=\left\{x \in U: \mu\left(B_{x}\right)>0\right\}
$$

has all the required properties. In the first place, $\mu\left(B_{x}\right)$ is a continuous function of $x_{1}, \ldots, x_{n}$ and hence of $x$, so that $V$ is an open subset of $U$. Further, let $x \in V$ so that $B_{x}$ is nonempty; if $y \in B_{x}$ then $y \in B$ and $x+T_{j} y \in A(j=1, \ldots, n)$; applying (7.13) it follows that $f_{0}$ is bounded below on $V$.

Finally, we have for almost all $x \in A$ that $x_{j}=T_{j}^{-1} x$ is a density point of the set $T_{j}^{-1} A(j=1, \ldots, n)$, so that 0 is a density point of $C_{x}$. But this in turn implies that $x \in V$ since $x \in A \subset U$ and $B$ has a positive upper density at 0 . 
(7.14) Let us now assume that $f$ is measurable. (Conjecture: this assumption is automatically satisfied.) We must show that $f_{0}$ is locally bounded below. We may assume that $T_{0}=0$ and that $U$ is bounded. Let $x_{0} \in U$ be given, and let $2 \delta$ denote its distance to the boundary of $U$. Let $W=\left\{x:\left|x-x_{0}\right|<\delta\right\}$.

Put $\delta \min _{j}\left\|T_{j}\right\|^{-1}=\varepsilon$ and $B^{\varepsilon}=\{y \in B:|y|<\varepsilon\}$. If $y \in B^{\varepsilon}$ then $\left|T_{j} y\right|<\delta$, hence, $x+T_{j} y \in U$ for each $x \in W,(j=1, \ldots, n)$. There further exists a measurable set $A \subset U$ such that $f$ is bounded on $A$ while the complement $A^{\prime}$ of $A$ in $U$ satisfies

$$
\mu\left(A^{\prime}\right) \sum_{j}\left|\operatorname{det}\left(T_{j}\right)\right|^{-1}<\mu\left(B^{\varepsilon}\right) .
$$

In order to prove that $f_{0}$ is bounded below on $W$ it suffices to show, by (7.13) with $T_{0}=0$, that for each $x \in W$ there exists $y \in B^{\varepsilon}$ such that $x+T_{j} y \in A(j=1, \ldots, n)$. If not then for some $x \in W$ and for each $y \in B^{\varepsilon}$ we have $x+T_{j} y \in A^{\prime}$ for some $j=1, \ldots, n$. This is easily seen to be impossible.

The following result is concerned with the "classical" case (7.1).

(7.15) THEOREM. Let $U$ be an open connected subset of $R^{p}$. Let $f$ be a function on $U$ which is bounded on some measurable subset $A$ of $U$ of positive measure.

Let $B$ be a nonempty open cone in $R^{p}$ with vertex 0 and suppose that $\Delta_{h}^{n} f(x) \geqq 0$ whenever $h \in B$ and $[x, x+n h] \subset U$; here, $n \geqq 2$. Then the function $f$ is continuous throughout $U$.

Proof. It suffices to consider the case that $U$ is convex. Let $E$ denote the set of points $x_{0} \in U$ at which $f$ is locally bounded. In view of Theorem 7.12, applied to (7.9), we have that $E$ is a nonempty open set.

On any straight segment $S$ in $U$ having a direction $h \in B$ the function $f$ is $(n-1)$ convex allowing us to employ the known one-dimensional results. In particular, we have from Theorem 2.2 that $f$ is continuous on $S$ as soon as $S \cap E$ is nonempty.

We shall first prove that $(E+B) \cap U \subset E$. Let $x_{0} \in E$ and $h \in B$ be such that $x_{0}+h \in U$; we must prove that $f$ is locally bounded at $x_{0}+h$. Let $V$ be a convex neighborhood of $x_{0}$ on which $f$ is bounded and consider $n$ equidistant hyperplanes $H_{i}$ perpendicular to $h$ which meet $V$. A line segment $S$ in the direction $h$ and sufficiently close to $x_{0}$ will meet $H_{i}$ in a point $x_{i}(i=1, \ldots, n)$. Considering the usual (one-dimensional) interpolating polynomial $L$ based on the set $\left\{x_{1}, \ldots, x_{n}\right\}$, it follows from (6.1) with $i=n$ that $f$ is locally bounded below at $x_{0}+h$, compare (4.4). In proving that $f$ is locally bounded above at $x_{0}+h$, consider the point $x_{n+1}=x_{0}+(1+\varepsilon) h \in U$ (for $\varepsilon>0$ sufficiently small) and draw straight lines through $x_{n+1}$ in directions $h^{\prime}$ sufficiently close to $h$ so that they will meet $H_{i}$ in a point $x_{i} \in V,(i=1, \ldots, n)$. Considering the interpolating polynomial based on the set $\left\{x_{1}, \ldots, x_{n-1}, x_{n+1}\right\}$, it follows from (6.1) with $i=n-1$ that $f$ is locally bounded above at $x_{0}+h$, compare (4.5). This proves our assertion.

In a similar way, using (6.1) with $i=0$ and $i=1$, one obtains that $(E-B) \cap U \subset E$. Any pair of points $x_{0}, y_{0}$ in $U$ can be connected by a chain $\left\{x_{k}, k=1, \ldots, N\right.$; $\left.x_{N}=y_{0}\right\}$ in $U$ such that $x_{k+1}-x_{k} \in B \cup(-B)$ for $k=0,1, \ldots, N-1$. It follows 
that $E=U$. In other words, $f$ is locally bounded throughout $U$, hence, by Theorem 2.2, $f$ is continuous along any straight line segment $S$ in a direction $h \in B$.

The usual proof of such continuity, see (4.6) and (4.9), applied to $S$ even yields uniform continuity of $f$ along $L$, at least locally near $x_{0}$. More precisely, given $x_{0} \in U$ there exists a convex neighborhood $V$ of $x_{0}, V \subset U$, such that, for each $\varepsilon>0$ there exists a positive number $\delta>0$ such that $|f(x+h)-f(x)|<\varepsilon$ as soon as $x \in V, h \in B \cup(-B),|h|<\delta$. Since $B$ is an open cone this in turn easily implies that $f$ is continuous at $x_{0}$.

(7.16) Assumption. From now on we shall restrict ourselves to the case $p=1$. Let $a_{0}, \ldots, a_{n}$ be given nonzero numbers satisfying (7.11). Let further $T_{0}=0<T_{1}$ $<\cdots<T_{n}$. Let $B$ be a given measurable subset of $[0,+\infty)$ having a positive density at 0 . Finally, let $f$ denote a real-valued function on the open interval $U=(a, b)$ in $R$ such that (7.10) holds whenever $y \in B$ and $a<x<b-T_{n} y$.

By the index $k$ of the relation (7.10) we shall mean the integer $k \geqq 0$ such that the numbers

$$
b_{r}=\sum_{j=0}^{n} a_{j} T_{j}^{r}, \quad(r=0,1, \ldots),
$$

satisfy $b_{r}=0$ for $0 \leqq r \leqq k$ and $b_{k+1} \neq 0$. In other words, $\sum_{j=0}^{n} a_{j} g\left(x+T_{j} y\right)=0$ holds for each polynomial of degree $\leqq k$ but not for all polynomials of degree $k+1$. Note that $k \leqq n-1$.

We shall also use the numbers defined by

$$
A_{j}=a_{j}+a_{j+1}+\cdots+a_{n}, \quad(j=0,1, \ldots, n) .
$$

Thus $A_{0}=0, A_{1}=-a_{0} \neq 0, A_{n}=a_{n} \neq 0$.

(7.18) THEOREM. Assume (7.16) and further that $f$ is measurable.

(I) If $k=0$ then $f$ is monotonic.

(II) If $f$ has at least one point of discontinuity then $k=0$ and either $A_{j} \geqq 0$ $(j=1, \ldots, n)$ or $A_{j} \leqq 0(j=1, \ldots, n)$. In all other cases $f$ is continuous throughout $(a, b)$.

(III) If $k \geqq 1$ then the derivative $f^{(k-1)}$ exists everywhere and admits the representation

$$
f^{(k-1)}(x)=f^{(k-1)}\left(x_{0}\right)+\int_{x_{0}}^{x} \phi(t) d t, \quad(\text { all } a<x<b),
$$

with $\varepsilon \phi$ as a nondecreasing function. Here, $\varepsilon=\operatorname{sgn}\left(b_{k+1}\right)= \pm 1$.

Observe that (III) implies the existence everywhere of a left continuous left derivative $f^{(k)}(x-0)=\phi(x-0)$ and a right continuous right derivative $f^{(k)}(x+0)$ $=\phi(x+0)$. Moreover, $f^{(k)}(x)$ exists except at the denumerably many points where $\phi$ makes a jump. It is also an easy consequence of (7.19) that $e f$ is a continuous $(k+1)$-convex function, compare (2.1). 
(7.20) Proof of Theorem 7.18. It follows from the second assertion of Theorem 7.12 that $f$ is locally bounded. Hence, $f$ defines a distribution $S$ in the sense of Schwartz [21].

Let $C^{\infty}$ denote the collection of all infinitely differerentiable functions $\psi$ on $(a, b)$ having a compact support $K_{\psi} \subset(a, b)$. Let $\psi \in C^{\infty}$ and let $y>0$ be so small that $K_{\Downarrow} \subset\left(a, b-T_{n} y\right)$. For such $y$ define

$$
G_{\psi}(y)=\sum_{j=0}^{n} a_{j} \int f\left(x+T_{j} y\right) \psi(x) d x=\sum_{j=0}^{n} a_{j} \int f(z) \psi\left(z-T_{j} y\right) d z .
$$

Clearly, near 0 the function $G_{\psi}(y)$ is infinitely differentiable with

$$
G_{\psi}^{(r)}(0)=b_{r}(-1)^{r} \int f(z) \psi^{(r)}(z) d z=b_{r} S^{(r)} \psi
$$

compare (7.17). Since $b_{r}=0$ for $0 \leqq r \leqq k, b_{k+1} \neq 0$ this implies that

$$
G_{\psi}(y) \sim c y^{k+1} S^{(k+1)} \psi \quad \text { for } y \downarrow 0,
$$

where $c=b_{k+1} /(k+1)$ !, $\operatorname{sgn}(c)=\operatorname{sgn}\left(b_{k+1}\right)=\varepsilon$.

Now consider $\psi \in C^{\infty}$ with $\psi \geqq 0$. Multiplying (7.10) by $\psi$ and integrating, we have that $G_{\psi}(y) \geqq 0$ when $y \in B, K_{\psi} \subset\left(a, b-T_{n} y\right),(y>0)$. It follows from (7.21) that

$$
\varepsilon S^{(k+1)} \psi \geqq 0 \quad \text { whenever } \psi \in C^{\infty}, \psi \geqq 0 .
$$

We now conclude, [21, p. 28, p. 54], that $\varepsilon S^{(k+1)}$ behaves as a nonnegative measure, while $\varepsilon S^{(k)}$ behaves as a nondecreasing $\phi$. It follows [21, p. 52], that $\varepsilon S$ behaves as a function of the form

$$
g(x)=P(x)+\int_{x_{0}}^{x} d t_{1} \int_{x_{0}}^{t_{1}} d t_{2} \cdots \int_{x_{0}}^{t_{k-1}} \phi\left(t_{k}\right) d t_{k} .
$$

Here, $P$ denotes a unique polynomial of degree $\leqq k-1$ (depending on $x_{0}$ ). For definiteness, let $\varepsilon=1$, that is, $b_{k+1}>0$. We now have that there exists a null set $N$ such set

$$
f(x)=g(x) \quad \text { if } x \notin N, a<x<b .
$$

If $k=0$ then $g=\phi$ is nondecreasing and $g(x-0) \leqq g(x) \leqq g(x+0)$. If $k \geqq 1$ then $g$ is continuous.

Let $a<x<b$ be fixed. Choose $0 \leqq i \leqq n$ fixed for the moment. For almost all $y \in B$ we have that $x+\left(T_{j}-T_{i}\right) y \notin N$ for all $j \neq i$. For such values $y$, using (7.10) (with $x$ replaced by $x-T_{i} y$ ) and (7.23), we have

$$
0 \leqq \sum_{j=0}^{n} a_{j} f\left(x+\left(T_{j}-T_{i}\right) y\right)=\sum_{j=0 ; j \neq i}^{n} a_{j} g\left(x+\left(T_{j}-T_{i}\right) y\right)+a_{i} f(x) .
$$


In other words, given $x$ and $i$, (7.24) holds for almost all $y \in B$ (sufficiently close to 0 ). Letting $y \downarrow 0$, this gives

$$
A_{i}[g(x+0)-g(x-0)]+a_{i}[f(x)-g(x+0)] \geqq 0,
$$

$(i=0,1, \ldots, n)$. If $g$ is continuous at $x$ we conclude from $(7.25)$ that $f(x)=g(x)$, since $a_{i}$ can have both signs. This yields assertion (III), and further (I) if $g$ is continuous everywhere.

Consider now the case that $k=0$ and that

$$
D=\{x: g(x-0)<g(x+0)\}
$$

is nonempty. Recall that $f(x)=g(x)$ if $x \notin D$. Applying (7.24) for points $x \notin D$, then letting $x \downarrow x_{0}$, and afterwards letting $y \downarrow 0(y \in B)$, one obtains that, for each point $x_{0} \in D$,

$$
A_{i}\left[g\left(x_{0}+0\right)-g\left(x_{0}-0\right)\right] \geqq 0, \quad(i=0, \ldots, n),
$$

hence, $A_{i} \geqq 0(i=0, \ldots, n)$. Thus $a_{0}=-A_{1}<0$ and $a_{n}=A_{n}>0$. Applying (7.25) with $i=0$ and $i=n$, we find that $g(x-0) \leqq f(x) \leqq g(x+0)$ for all $x$, so that $f$ is monotonic.

By the way, in any case where $A_{i} \geqq 0$ for all $i$ we must have $k=0$, and (7.10) holds for any nondecreasing function $f$, as follows from a simple summation by parts.

\section{REFERENCES}

1. E. F. Beckenbach and R. H. Bing, On generalized convex functions, Trans. Amer. Math. Soc. 58 (1945), 220-230.

2. P. L. Butzer and W. Kozakiewicz, On the Riemann derivatives for integrable functions, Canad. J. Math. 6 (1954), 572-581.

3. Z. Ciesielski, Some properties of convex functions of higher orders, Ann. Polon. Math. (Warsaw) 7 (1959), 1-7.

4. A. Denjoy, Sur l'intégration dẹs coefficients différentiels d'ordre supérieur, Fund. Math. 25 (1935), 272-326.

5. P. Halmos, Measure theory, Van Nostrand, New York, 1950.

6. P. Hartman, Unrestricted n-parameter families, Rend. Circ. Mat. Palermo (2) 7 (1958), 123-142.

7. S. Karlin and W. J. Studden, Tchebycheff systems: with applications in analysis and statistics, Interscience, New York, 1966.

8. J. L. Kelley, General topology, Van Nostrand, New York, 1955.

9. S. Kurepa, A property of a set of positive measure and its application, J. Math. Soc. Japan 13 (1961), 13-19.

10. E. Moldovan, Sur une généralisation des fonctions convexes, Mathematica (Cluj) (2) 1 (1959), 49-80.

11. P. Montel, Sur les polynomes d'approximation, Bull. Soc. Math. France 46(1918), 151-192.

12. M. I. Morozov, On some problems on the uniform approximation of a continuous function by means of functions in an interpolation class, Izv. Akad. Nauk SSSR Ser. Mat. 16 (1952), 75-100. (Russian) 
13. A. Ostrowski, Über die Funktionalgleichung der Exponentialfunktion und verwandte Funktionalgleichungen, Jber. Deutsch. Math.-Verein. 38 (1929), 54-62.

14. M. M. Peixoto, On the existence of a derivative of generalized convex functions, Summa Brasil. Math. 2 (1948), 35-42.

15. G. Pólya, On the mean value theorem corresponding to a given linear homogeneous differential equation, Trans. Amer. Math. Soc. 24 (1922), 312-324.

16. G. Pólya and G. Szegö, Aufgaben und Lehrsätze aus der Analysis. II, Springer-Verlag, Berlin, 1925.

17. T. Popoviciu, Sur quelques propriétés des fonctions d'une ou de deux variables réelles, Mathematica (Cluj) 8 (1934), 1-85.

18. _ - Notes sur les fonctions convexes d'ordre superieur (I), Mathematica (Cluj) 12 (1936), 81-92.

19. - Les fonctions convexes, Actualités Sci. Indust., No. 992, Hermann, Paris, 1944.

20. — Sur le reste dans certaines formules linéaires d'approximation de l'analyse, Mathematica (Cluj) (2) 1 (1959), 95-142.

21. L. Schwartz, Théorie des distributions, Vol. I, Actualités Sci. Indust., No. 1091, Hermann, Paris, 1950.

22. L. Tornheim, On n-parameter families of functions and associated convex functions, Trans. Amer. Math. Soc. 69 (1950), 457-467.

23. O. Haupt and H. Künneth, Geometrische Ordnungen, Springer-Verlag, New York, 1967.

24. Z. Nehari, Disconjugate linear differential operators, Trans. Amer. Math. Soc. 129 (1967), $500-516$.

UNIVERSITY OF ROCHESTER,

ROCHESTER, NEW YORK 